\title{
Parametric Optimization Design of Brake Block Based on Reverse Engineering
}

\author{
JIN Hua-wei ${ }^{1, a}$, WANG Zhe ${ }^{1}$, ZHANG Xin ${ }^{1}$ and WANG Sai ${ }^{1}$ \\ ${ }^{1}$ Mechanical Engineering School, Anhui University of Science and Technology, Huainan, 232001, China
}

\begin{abstract}
As one of the key part of automotive brake, the performance of brake block has a direct impact on the safety and comfort of cars. Modeling the brake block of disc brake in reverse parameterization by reverse engineering software, analyzing and optimizing the reconstructed model by CAE software. Processing the scanned point cloud by Geomagic Studio and reconstructing the CAD model of the brake block with the parametric surface function of the software, then analyzing and optimizing it by Wrokbench. The example shows that it is quick to reconstruct the CAD model of parts by using reverse parameterization method and reduce part re-design development cycle significantly.
\end{abstract}

\section{Introduction}

Reverse engineering is technology, which choosing advanced production equipment, sample piece, software (including drawings, procedures, technical documents or images and so on) as the research object applies the modern design method, production engineering, materials science and relevant professional knowledge to System analysis and research, and to explore and master the key technology in order to develop more advanced products of the same kind, developed in 1990s. Geomagic Studio is an automated reverse engineering software, which can automatically generate accurate digital models through scanning point cloud[1]. Geomagic Studio can output industry standard formats, including STL, IGES, STEP and CAD and many other file formats. Parametric reconstruction model can fast analysis of product design intent and redesign the product.

Brake block is one of the carrier of automobile brake, which directly affects the braking performance and comfort level. The brake block is divided into the friction block and the brake back plate and the friction force between the friction block and the brake disc is used to eliminate the kinetic energy of the vehicle, so as to achieve the purpose of braking. The structure of the brake block has a critical influence on the stress distribution and contact state, so it indicate that analyze the brake block by finite element method would be meaningful. After working for a period time,it is inevitably that cause brake block wear and other missing. In this case, it is difficult to realize the optimal design of the brake block only by the forward modeling software. In this article,through three dimensional scanning of the wear disc brake block can get the point cloud data.By Geomagic Studio make a parametric curved surface structure, and reestablish the three dimensional CAD model. The resulting model is analyzed by ANSYS Workbench for stress and contact analysis and optimized.

a JIN Hua-wei (1986-),E-mail: anlijinhuawei@126.com. 


\section{Parameterized reconstruction of model based on Geomagic Studio}

\subsection{Point cloud data acquisition and processing}

In this paper, the point cloud acquisition device is Tianjin Weishen Technology Vtop 130 threedimensional scanner, which scanning method is non-contact and single scanning accuracy is $0.03 \mathrm{~mm}$. the point cloud data of scanning is shown in Figure 1, then input the resulting point cloud into the Geomagic Studio software to reduce the noise of the point cloud data and then packaged into the polygon processing phase. In order to facilitate the parameterized after reconstruction, at this time the polygon data are divided into two parts and handle separately.After filling holes,adjusting and repairing mesh to be finished will get the triangular surface shown in Figure 2.

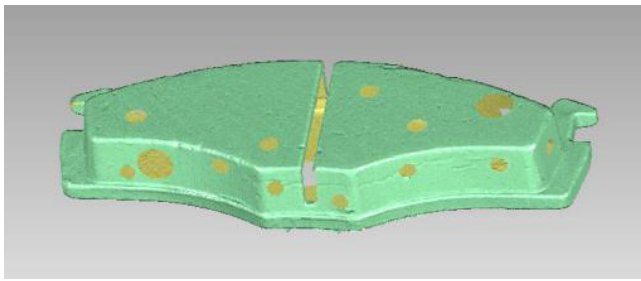

Figure 1. Point cloud data

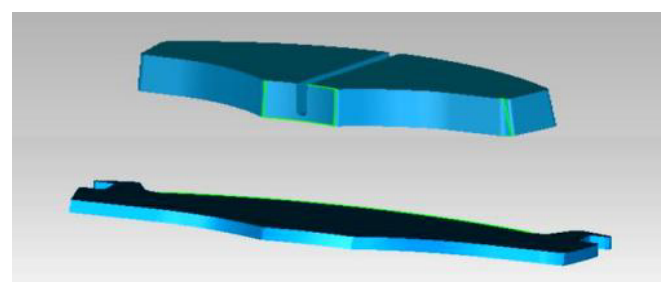

Figure 2. Triangular surface

\subsection{Constructing parametric surface and reconstructing model}

After the processing of the polygon phase, it accesses Geomagic Studio stage of parametric surfaces. Surface reconstruction is an important stage of reverse engineering. After the formation of the curved surface, the model will form a uniform size of the surface patches.Then, through the software function of structural grid, we can form a uniform grid network on the surface of the model, the more uniform the grid, the more smooth the surface.Finally, the Geomagic Studio will automatically synthesize the NURBS surface on the basis of the grid network and form the required model [2]. After cutting and stitching, The CAD model shown in Figure 3 , and output STEP or other common format file

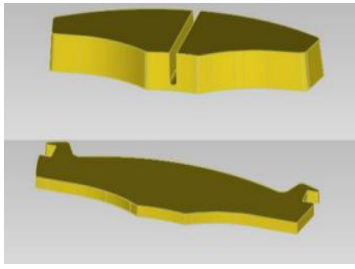

Figure 3. CAD model

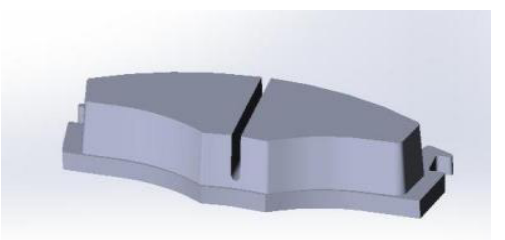

Figure 4. Reconstruction model of brake block

\section{Finite element analysis and optimization of brake block}

\subsection{Coordination of upper and lower parts and model processing}

Generally speaking, parts obtained by CAD software need a common data format to import professional CAE software, but through this conversion may make the original file data missing, resulting in broken surface and other issues, and direct affect the subsequent CAE analysis[3]. The IGS format files from Geomagic Studio need to be imported into Solidworks for input diagnosis repair and Recognition feature,mean while Solidworks can directly exchange data with ANSYS and Workbench, to avoid data loss. After Solidworks processing to get the final reconstruction of the CAD model shown in Figure 4. 


\subsection{Stress analysis and model definition of brake block}

In the process of braking, due to the limit of the brake guide groove on both sides of the back brake block, the brake block plate can only slide along the axial direction, while the piston exerts an axial force on the brake backing under the action of hydraulic pressure, which causes the brake block and brake disk contact, and generate a braking force. In this paper, the contact between brake pads and brake discs is studied, Ignoring the effect of friction on contact analysis. Compared with the brake disc, the stiffness of the friction block is relatively small, and for simplicity of analysis, the brake disc is reduced to a rigid surface.

After importing the rebuilt model into ANSYS Workbench, it is necessary to define the material and connection. The density of the friction block is $2615 \mathrm{~kg} / \mathrm{m} 3$, the elastic modulus is $8600 \mathrm{MPa}$, the Poisson's ratio is 0.3 ; the density of the brake pad is $7800 \mathrm{~kg} / \mathrm{m} 3$, the elastic modulus is $182000 \mathrm{MPa}$, the Poisson's ratio is 0.3 .The connection between the friction block and the brake backplane is defined as "bonded" and the connection between the friction block and the rigid face is defined as "rough" and the rigid face is the target face.

\subsection{Mesh generation}

In the process of finite element analysis, meshing is the key step in finite element analysis. The accuracy and efficiency of finite element analysis are directly affected by meshing. Hexahedral element is more accurate and has better mechanical properties than the tetrahedral element[4-5]. According to the brake backplane choose the method of multi body partition, mapping division selection of hexahedron. The friction block adopts hexahedral partition method, the overall accuracy is $1.5 \mathrm{~mm}$.After the completion of the mesh shown in Figure 5.

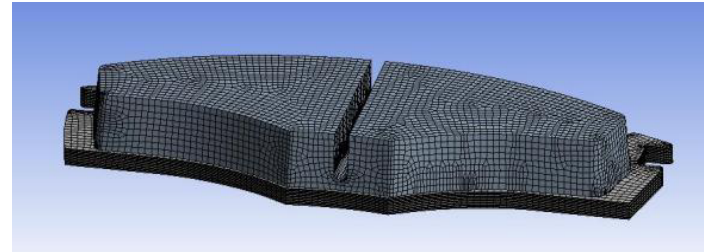

Figure 5. Brake Block Meshing

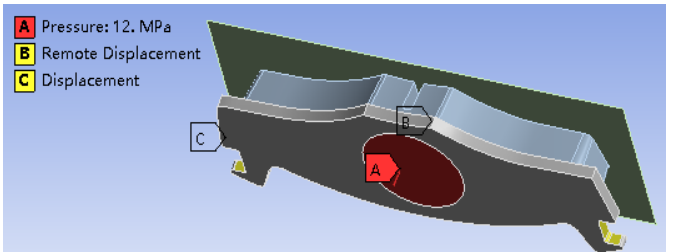

Figure 6. The application of boundary conditions

\subsection{Applied loads and boundary conditions}

According to the actual working condition, the $12 \mathrm{MPa}$ pressure is applied in the brake backplane center diameter of $35 \mathrm{~mm}$ circular area; In the axial direction of the $\mathrm{Z}$ axis, in the back of the brake guide groove to apply $\mathrm{X}$ direction and the $\mathrm{Y}$ direction of the fixed displacement constraints, $\mathrm{Z}$ direction of freedom; The rigid surface is applied a remote displacement constraint, the $X$ direction, the $\mathrm{Y}$ direction, the $\mathrm{Z}$ direction, and the rotation around the $\mathrm{X}$ axis, the $\mathrm{Y}$ axis is rotated, and the $\mathrm{Z}$ axis is set to be fixed. Open the large deformation option "Large Deflection" in the analysis settings. The result of the application is shown in figure 6 .

\subsection{Analyze options and results}

The maximum stress point can be seen through the equivalent stress cloud as shown in Figure 7, and the maximum is $55.231 \mathrm{MPa}$; The maximum stress point between the friction block and the brake disc is shown in Fig. 8, and the maximum value is $11.345 \mathrm{MPa}$; The contact state between the friction block and the brake disc is shown in figure 9. 


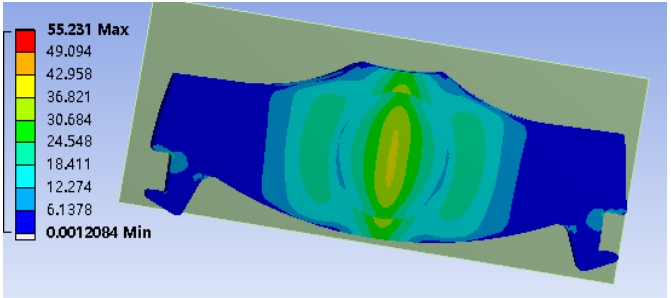

Figure 7. Equivalent stress

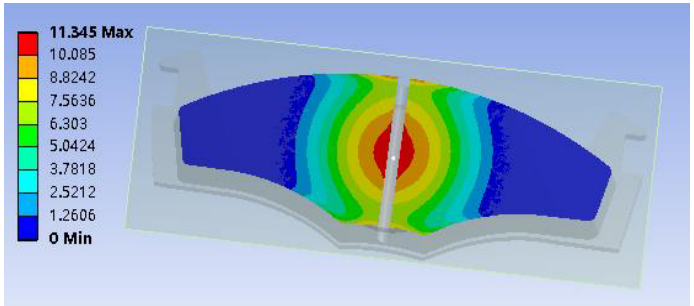

Figure 8. Contact stress

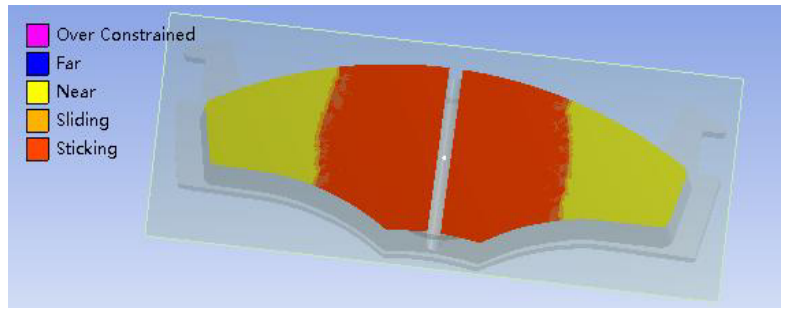

Figure 9. Contact state

\section{Optimization of brake block structure}

\subsection{The scheme determination of optimization}

The 3D model reconstructed in Geomagic Studio can not be optimized directly using ANSYS Workbench, which needs to be imported into Solidworks, and add the ds_ prefix to the parameter name that needs to be optimized[6]. The above analysis shows that there are more non contact surfaces on both sides of the friction block with the brake disc, and the brake block equivalent stress, will make the brake block wear uneven and affect the service life.The shape of the friction block, the shape and thickness of the brake back plate will cause the change of contact state and contact pressure.In this paper, the influence of the thickness of the brake pad on the contact state is studied.The input parameters are the thickness of the brake back plate, and the output parameters are the equivalent stress and contact stress of the brake block. Set the original thickness of the brake back plate is $4 \mathrm{~mm}$, the optimization range is set to $3.4 \mathrm{~mm}$ to $4.6 \mathrm{~mm}$, divided into five design points.

\subsection{Optimization results and analysis}

Through analysis and calculation, the input and output parameters of the 5 design points are shown in table 1.It can be seen that when the thickness of the brake plate is less than the original size, the contact stress is relatively increased, but the maximum equivalent stress is relatively reduced; when the thickness of the brake plate is larger than the original size, the contact stress is relatively reduced and the maximum equivalent stress is also reduced slightly.

Table 1. Design point input and output parameters

\begin{tabular}{|c|c|c|c|}
\hline Serial number & $\begin{array}{c}\text { Brake backing thickness } \\
(\mathrm{mm})\end{array}$ & $\begin{array}{c}\text { Maximum contact stress } \\
(\mathrm{MPa})\end{array}$ & $\begin{array}{c}\text { Maximum equivalent } \\
\text { stress }(\mathrm{MPa})\end{array}$ \\
\hline 1 & 3.4 & 11.963 & 55.399 \\
\hline 2 & 3.7 & 11.653 & 50.515 \\
\hline 3 & 4.0 & 11.345 & 55.231 \\
\hline
\end{tabular}




\begin{tabular}{|l|l|l|l|}
\hline 4 & 4.3 & 11.035 & 53.020 \\
\hline 5 & 4.6 & 10.768 & 52.255 \\
\hline
\end{tabular}

To ensure that the contact state, should make the contact stress to optimize the highest priority, and for the convenience of processing should select thickness of $4.5 \mathrm{~mm}$. Choosing the optimal size set to the current design point, the optimized brake block equivalent stress nephogram after re-calculated shown in Figure 10, the maximum equivalent stress of $51.592 \mathrm{MPa}$; The contact stress cloud is shown in Figure 11, the maximum contact stress is $10.866 \mathrm{MPa}$; contact state diagram shown in Figure 12.

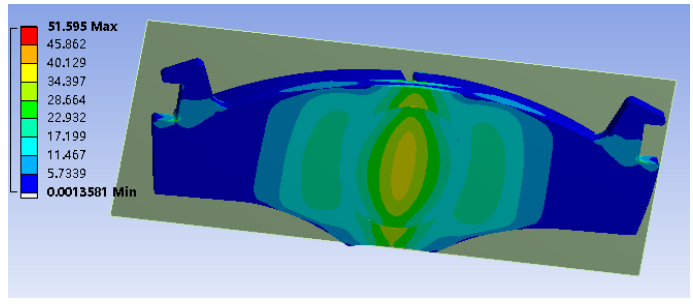

Figure 10. equivalent stress after optimization

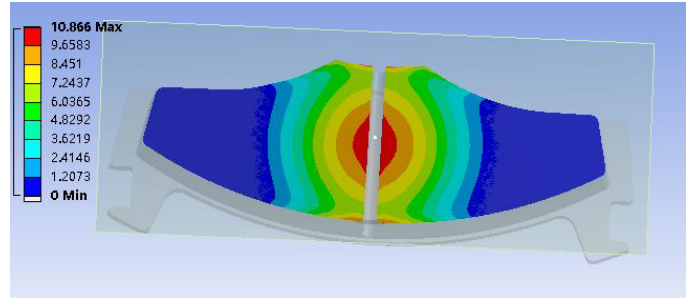

Figure 11. contact stress after optimization

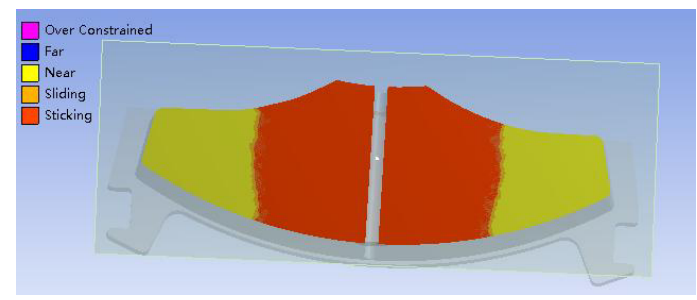

Figure 12. Optimized contact state

The optimized maximum contact stress, maximum equivalent stress is compared with that before optimization, as shown in Table 2. It can be seen that the maximum contact stress after optimization is reduced by $0.479 \mathrm{MPa}$ and the maximum equivalent stress is reduced by $3.639 \mathrm{MPa}$.

Table 2 . Comparison before and after optimization

\begin{tabular}{|c|c|c|c|}
\hline & $\begin{array}{c}\text { Brake backing thickness } \\
(\mathrm{mm})\end{array}$ & $\begin{array}{c}\text { Maximum contact stress } \\
(\mathrm{MPa})\end{array}$ & $\begin{array}{c}\text { Maximum equivalent } \\
\text { stress }(\mathrm{MPa})\end{array}$ \\
\hline $\begin{array}{c}\text { Pre-optimization } \\
\text { values }\end{array}$ & 4.0 & 11.345 & 55.231 \\
\hline Optimized value & 4.5 & 10.866 & 51.592 \\
\hline Change value & & -0.479 & -3.639 \\
\hline Optimization rate & & $4.2 \%$ & $6.6 \%$ \\
\hline
\end{tabular}

\section{Conclusion}

The results show that the real model and parameters can be quickly restored by the reverse engineering software, and the parameters of the model can be reconstructed quickly, especially in the case of parts with wear or other defects, compared with single forward modeling, and it can be re- 
designed with most of the forward modeling software. Through this method, the development process of parts re-design can be greatly reduced and the market competitiveness of the products can be improved.

\section{Acknowledgements}

This research is supported by the key project of Anhui Province Natural Science Foundation(Working mechanism and structure research of automotive micro electrical and mechanical brake device), the project of Anhui Province Science and Technology Research Project (1301022074,1301022075), the key project of Anhui Province Natural Science Foundation (No.KJ2015ZD019) and the key project of Young Teachers' Scientific Research Fund of Anhui University of Science and Technology (QN201416).

\section{References}

1. JinTao,Tong Yong-guang. Reverse Engineering Technology[M].BeiJing.China Machine Press.(8):1-5 (2003).

2. JuLin-guang,Bian Xianhu,Heavy truck bumper Geomagic Catia software and the drag hook gate number mode reconstruction[J]. machinery. based on the.39 (4):6 10 (2012).

3. ChouCan-hua,ChengSi-yuan,ZhangXiang-wei,and so on. Finite element analysis of parts based on reverse engineering technology[J]. Machinery Design and Manufacture.(9):35-36 (2009).

4. Che X,Liang X,Li Q.G1 continuity conditions of adja - cent NURBS surfaces[J].Computer Aided Geometric De.sign.22(4):285-298 (2005).

5. Shi X.Wang T,Wang T,et a1.Reconstruction of convergent G1 smooth B-spline surface[J]. Computer Aided Geometric Design.21(9):893 - 913 (2004).

6. HanJiang,MengChao, YaoYin-ge, and so on.Parametric optimization design based on Reverse Engineering[J]. Combined machine tool and automatic machining technology.(3):38-39 (2016). 